\title{
The potential of co-creation spaces to contribute to Sustainable Development
}

\author{
Collaborative production in community-based \\ open workshops has a potential to contribute \\ to sustainable development. The article explores \\ how open workshops, collaborative innovation \\ and manufacturing can contribute to sustain- \\ ability of a city such as Berlin. \\ By Alexandr Iscenco
}

identify the details and implications of this connection. From one side, open workshops offer the potential of saving and reusing natural resources, localizing production of goods, reducing greenhouse-gas-emitting transportation throughout the value chain of a product, and stimulating crowdsourced and openly accessible eco-innovations. From another side, however, such a trend might lead to the increase of wasteful production and consumption and higher demand for energy that may come from unsustainable sources. Thus, the research question explored in the present article is how the collaborative innovation and production in open workshops can be connected to sustainable development of a city.

\section{Introduction}

The Maker Movement, or the Third Industrial Revolution, as it is sometimes called, lies in the fact that individuals become a significant influencing force on their own production and consumption of physical goods. The global distribution and accessibility of the Web, and desktop manufacturing technologies available openly nowadays, allow practically anyone to not only produce and customize their own goods, but also to engage in entrepreneurial manufacturing activity with minimum investments and efforts required. This empowers individuals to innovate and compete in production even with the global manufacturing giants in various fields of economic activity. As Anderson (2012) states, the Maker Movement gives hardware entrepreneurs the ability to be both, small and global.

One of the most evident manifestations of the Maker Movement is the collaborative innovation, prototyping, and manufacturing of hardware in community-oriented co-creation spaces often referred to as open workshops (Verbund Offener Werkstätten 2014). These include co-working spaces, hackerspaces, makerspaces, fab labs, and other workshops. They offer an individual the possibility to openly access production tools and machines, specifically 3D printers, Computerized Numerical Control (CNC) mills, and laser cutters, and use them for prototyping and manufacturing of hardware goods in a space shared with other like-minded software and hardware enthusiasts and entrepreneurs, who call themselves hackers and makers. The creations coming from open workshops can range from simple 3D printed pendants and other accessories to complex Arduino-based robots, drones, and even whole 3D printed and assembled cars (Business Insider 2014).

One can discern the potential connection between sustainable development and the Maker Movement manifesting itself in co-creation in open workshops. However, it is a challenge to

\section{Open workshops and their types}

\subsection{Definition}

There are a number of definitions of open or collaborative workshops. For example, Verbund Offener Werkstätten (2014) describes them as open collaborative spaces with equipment and tools available to all technically and artistically active individuals (hackers, makers, crafters, tinkerers, and artists) and their groups allowing them to work on their software, hardware, or art projects. In open workshops these people can collectively build or repair devices, design inventions, manufacture prototypes, sew clothes or produce works of art. Thus, the focus here is on collective and creative actions by creative and innovative people coming together.

The definition of open workshops given by Kostakis et al. (2014) describes them as physical places working on the basis of the community created around them, where individuals are immersed in the culture and ethics of each place and are able to engage with meaningful and creative projects. Thus, the authors stress the community basis of every workshop and the specific environment and ethics present in it.

Nowadays there is no commonly established and agreed upon definition of an open workshop (Moilanen 2012). The reason for the challenge in coming up with one lies in large heterogeneity of such places. It means that open workshops are so diverse that it is practically impossible to find two workshops that are alike, even though they are of the same type (Lobo 2011).

Still, in spite of all the differences, there are some common features of open workshops that unite them and distinguish them from other economic agents. These are:

- Open workshops are all real physical places, where people can come and work on, and contribute to collaborative projects in person. 
- Blurred organizational borders between professional, semiprofessional, and non-professional ways to co-create and innovate. It indicates that open workshops welcome both amateurs and professionals.

- Existence of intrinsic positive motivation of open workshop members to create projects within a community formed around the workshop.

- Promotion of collaborative relations and common accountability based on mutual trust among members of open workshops.

- Focus on independence and autonomy of open workshops; that is, they should be self-sustaining entities not belonging to a specific company or governmental body.

- Community-driven functioning of open-workshops. Such places are indeed independent, but they are never standalone entities. Every workshop is connected to a community and network of other workshops of similar type.

\subsection{Classification}

Open workshops can also be classified into different types based on the combination of common features and distinctive characteristics listed above. As in the case of their definition, there is also no single common classification available. The challenge is the same: significant differences in ownership, management, activities or accessibility between the workshops.

The most well-known classification groups open workshops into hackerspaces, makerspaces, tech shops, and fab labs (Cavalcanti 2013; Lang 2013). However, the present research showed that one can identify ten distinctive types of open workshops:

- Co-working space is a professional or semi-professional space for any type of work. Mostly it is used as shared space for entrepreneurs, startup teams, and small businesses.

- Hackerspace is a grassroots community-operated workspace, where people with common interests into hacking or making co-create (Parker 2013) [1]. It is considered to be the original type of maker community space that maintains its focus mostly on repurposing hardware, working on electronics, and programming software (Hackerspaces.org 2013; Lang 2013).

- Makerspace is usually considered as a more professional and more mainstream subcategory of hackerspace. The term defines a publicly-accessible place to design, craft, and create physical objects and hardware solutions (Cavalcanti 2013). Makerspace often uses a business-oriented approach through fee-based membership. However, it is usually registered as a non-profit entity reinvesting all its revenue in the development of the place.

- Maker Faire stands for a community-organized event for makers, crafters, and tinkerers to show their creations to other makers and the general public, as well as hack or make together with the visitors at the place of the event. Maker Faire can be considered a temporarily or periodically organized and openly accessible combination of a makerspace and showroom.
- Maker Lab is another maker-focused temporary event. The difference between Maker Faire and Maker Lab is that the former is organized mostly by a community and is aimed at showing the creations of the makers, while the latter is the product of the efforts of a certain individual or team and is oriented towards educating interested people about hacking or making.

- Fab Lab is the permanent version of a Maker Lab. It is a small-scale real-life laboratory offering digital fabrication tools. Fab Lab can be considered the educational take on making powerful production tools more accessible (Cavalcanti 2013). Such places are often registered as, and run by, local non-profit organizations. Fab Labs operate according to the Fab Charter that can be found on the MIT (Massachusetts Institute of Technology) website (MIT's Center for Bits and Atoms 2007).

- Tech Shop is the for-profit business variation of a makerspace aimed at offering professional tools and machines for rent (Cavalcanti 2013). It gets its revenue from fee-based classes and workshops on making or hacking and renting of well-kept professional tools and high-end equipment infrastructure (Lang 2013).

- Specialized Workshop is what one can call a specialized craft- and creativity-based co-creation space with specific focus on certain aspects of making and craftsmanship. There are workshops on knitting, sewing, pottery, painting, filmmaking, electronics, etc. Often they have certain educational elements, such as fee-based classes and workshops.

- 3D Printing Café represents a for-profit combination of a café with food and drinks and a 3D printing workshop equipped with a variety of 3D printers, filaments for them, and tools for cleaning 3D creations. It offers both 3D printing services and 3D printing educational activities for a price.

- Repair Café or Repair Shop is another combination of a café and an open workshop. Only in this case it is focused on repairing broken things and devices by the visitors themselves under the guidance of skillful and experienced makers.

Nowadays co-working spaces, hacker- and makerspaces as well as 3D printing cafés represent the majority of existing open workshops. The main reason is that it is relatively easier to set up such places than other types (Cavalcanti 2013).

\section{Connection between open workshops and sustainability}

Collaborative activity and the sharing principle of open workshops offer significant potential to contribute to sustainable development of a city and country as a whole. The most common arguments supporting this point of view are the following:

- Reduction of greenhouse gas (GHG) emissions from manufacturing and transportation of products, since each product is made locally in an open workshop and thus made available to the end consumer at the same place (Iscenco 2014). 
I Increased resource efficiency and reduced waste generation due to the fact that modern machines in open workshops use no more raw materials than they need. For instance, 3D printing software is designed to calculate how to print an object with the least amount of filament possible. In fully enclosed designs it orders the 3D printer to create a honeycomb structure to save the raw material and at the same time make the whole creation rigid. In addition to that, the makers, crafters and artists in open workshops use and share the same tools and resources among each other (Anderson 2012).

I Another potential contribution of open workshops to the reduction of waste lies in the fact that they give opportunity to create personalized products. And such products tend to be less disposable, since their owners simply care about them more than conventional serial goods and therefore try to use them as long as possible (Anderson 2012; Iscenco 2014).

- Shift away from overconsumption, planned obsolescence, and disposability of goods. Projects created by a community in an open workshop are designed to last longer and be constantly improved (Kostakis et al. 2014).

I Reduction of environmental footprint of each product coming out of open workshops. Co-creation in such places and the Maker Movement in general are based on sharing of open knowledge, designs, and manufactured creations. And sharing the created goods does not diminish their value, but actually enhances it, while at the same time decreases their environmental footprint. Thus, one resource or good creates multiple value and use during its life cycle (Kostakis et al. 2014).

I There are projects and initiatives to integrate the circular flow of materials in the activity of open workshops. One of these initiatives focuses on circular resource flow in 3D printing through designing and using a special device that can shred plastic and transform it into filament for 3D printers. Such a device, a FilaMaker, is already available as an open-source design and ready-made product for sale (FilaMaker 2014; Iscenco 2014).

I Finally, certain workshops have started to transform into environmentally-friendly ones. Green Fab Labs are beginning to appear on the maker scene worldwide. Besides the Fab Charter, they also integrate the principles of circular resource flow and minimization of environmental footprints (Iscenco 2014).

However, in spite of all the arguments supporting the potential of open workshops to contribute to sustainable development, one can also conceive of the potentially negative effects of these places on sustainability and specifically on the environment. These can include:

- The relative ease and freedom of personal manufacturing may result in overproduction of unnecessary items and without the efficient recycling of the infrastructure, even more waste is generated (Iscenco 2014).
I Modern desktop manufacturing machines such as 3D printers, CNC mills, and laser cutters, require large amounts of energy to operate. This places additional burden on energy production in the area and additional greenhouse gas emissions if the energy needed is produced in traditional polluting ways.

- Local fabrication of goods in an open workshop does not guarantee their usage in the local area. With the global coverage of the Web an individual can order the manufacturing of his design anywhere in the world. For instance, such companies as Shapeways and Ponoko offer 3D printing of customer's designs along with shipment of the creations to the customer's address (Anderson 2012). This can involve large distances and significant GHG emissions from transportation.

All in all, there are both advocates and critics of collaborative fabrication in open workshops in terms of its relation to sustainability. The question as to whether co-creation spaces and their activities can contribute to sustainable development of a city or country remains open. In order to answer it, a research study involving open workshops in Berlin, Germany, was conducted.

\section{Collaborative sustainability in open workshops in Berlin}

\subsection{Purpose and objectives of the research}

The research entitled Collaborative Sustainability in Open Workshops was done from August to October 2014 in Berlin, Germany. Its main goal was to answer the following question: How can the collaborative innovation and commons-based peer production in open workshops be linked to sustainable development of a city, i. e., to its prosperity ecologically, economically, and socially?

Berlin was chosen as the research location due to the city being one of the key hotspots of the Maker Movement. This is the place where the first hacker- and makerspaces in Europe, such as c-base, were established. Additionally, it is where collaborative production and consumption, based on the maker culture, are developing in a comparatively fast way.

\subsection{Methodology}

The research work was based on study visits to a number of open workshops of different types in Berlin. Both off-line interviews and off-line surveying were done with the founders, organizers, and managers of a total of twelve open workshops: - Co-working spaces: co.up, MachWERK - Moabiter Werkstätten, and Impact Hub Berlin

- Hackerspace: c-base and 3D Berlin Headquarter/YOUin3D

- Makerspace: Betahaus and Open Design City

- Fab lab: Fab Lab Berlin

- Specialized workshop: Nadelwald Co-Sewing Space

I 3D Printing Café: Dimension Alley and Teptec Studios

- Repair Café: Repair Café Berlin-Schöneberg/BUND Berlin. 


\subsection{Analysis and discussion}

The research brought a number of interesting observations and valuable inputs. First of all, none of the representatives of twelve open workshops interviewed indicated sustainability, resource efficiency, social well-being, economic prosperity, or environmental friendliness among the reasons for establishing and running each particular workshop. The main motives here are giving shared access to the internet or tools, applying one's professional occupation and experience in hacking or making to running one's own entity, and doing something a founder likes and is passionate about.

This output indicates that nowadays open workshops are not sustainability-focused economic agents. Instead, they are private or community-based spaces oriented towards achieving personal or professional goals or sharing tools and access to the web.

Still, open workshops do involuntarily contribute to sustainable development in some ways, as further survey results show. The results are presented according to the three pillars of sustainability, or the Triple Bottom Line (The Economist 2009): economic growth (profit), social well-being (people), and environmental responsibility (planet).

In terms of economic development open workshops are divided into for-profit and non-profit entities. Among the twelve spaces surveyed only two are functioning as for-profit companies. However, even the non-profit ones generate some revenue that comes mainly from membership fees, fees for using tools and machines, payments for educational workshops, and additional services for corporate projects. Nevertheless, $54 \%$ of the respondents indicated that ensuring financial sustainability represents the main challenge for their co-creation spaces. The ways they use to overcome this challenge include taking on additional projects as paid services for corporate customers and collecting donations.

At the same time all open workshops surveyed try to stay independent and operate autonomously without any corporate or governmental support that might limit their free hacking or making culture and open-source focus of their projects. However, they do accept donations of tools, machines, and other non-restricting support from other economic agents. For instance at Fab Lab Berlin, the 3D printer MakerBot Replicator 2 was donated by the company MakerBot that produces them.

Overall, the contribution of open workshops to economic development of a city is limited because such places themselves are not designed to be the generators of significant economic welfare and growth from the start. The projects created within open workshops may well be the profitable ones, thus allowing these places to contribute to economic development indirectly. However, the survey was not able to elicit such information, as hacker- or makerspaces usually do not collect data on the projects created within them that went to the market.

In terms of social well-being the potential and positive impact of co-creation spaces is much more evident. According to the survey results, an open workshop can host up to 10 to 15 hackers or makers per day and have approximately 500 members per year. For example, c-base, one of the most well-known and developed hackerspaces not only in Berlin but in Europe as a whole, had 515 members when we surveyed them. Open workshops also have open days where they invite non-member visitors to explore their places, try the machines, and learn about hacking or making.

Such social engagement and networking opportunities nurture a strong community of passionate hackers or makers, allowing them to exchange knowledge, resources, and tools in a fast and efficient way. Furthermore, most of the projects in open workshops are created on a collaborative basis through the skill- and effort-based economy, where the currency is the knowledge, skills, and time of every workshop member. As a result, each project is done with minimal costs and effort from its creator while still maximizing its value for society.

An open workshop itself can create certain social value. The respondents of the survey indicated that the main reasons for people to come to their open workshops and stay there are the possibility to work on individual projects, get open access to diverse tools and machines that they cannot afford or do not want to purchase, learn about different aspects of hacking, networking with like-minded people, and sharing common interests in hacking with them.

These survey outputs point out an important role of open workshops in society. They serve as an openly accessible opportunity for citizens to increase their social well-being by creating their own goods and earning project-based additional income through using shared spaces and machines at a minimum cost to them. Co-creation spaces also help people connect with each other and engage in productive networking both within the communities around these spaces and outside of them. This happens because hacker- and makerspaces as well as fab labs usually interact with other open workshops outside the city and even the country. For example, members of c-base and 3D Berlin Headquarter/YOUin3D hackerspaces mentioned that they often participate at the Chaos Communication Congress, a large annual international meeting of hackers taking place in Hamburg, where they meet more than 9,000 like-minded people passionate about hacking from all over the world.

In terms of environmental responsibility and conservation of the natural environment there is potential for open workshops to contribute to these due to sharing of tools and machines, collaborative creation of local goods and thus minimization of waste and greenhouse gas emissions. However, the data on this aspect of sustainability in co-creation spaces are very limited, and the survey did not succeed in enriching that data significantly, as none of the spaces surveyed does any assessment of its own environmental footprint and tracking of eco-innovations created and implemented within them.

Still, certain open workshops do help reduce the amount of waste by recycling or repurposing it. For instance, in the case of Fab Lab Berlin some plastic waste from their 3D printers is collected and recycled into filament for these machines by a 
neighboring company, thus creating a closed-loop material flow in 3D printing there. Another good example is c-base where different waste items, especially waste electrical and electronic equipment, or e-waste, are collected, stored in a special room, and then reused by hackerspace members either in their own projects or as creative-plus-functional decorations in the hackerspace itself for it to look and function as a " 4.5 billion year old space station", as the legend about founding that place suggests (c-base 2014). Similar approaches are used in other hackerspaces, where e-waste becomes valuable as a free resource for projects.

To sum up, nowadays open workshops are not designed specifically to contribute to sustainable development and do not assess their impact based on economic, social, and environmental aspects of it. Still, involuntarily and indirectly they do have positive impact on all these aspects through offering people access to shared space, tools, machines, and a network for creating their own local goods and earning certain income from software and hardware projects in a resource-efficient and wasteminimizing way. And this impact will continue to increase as the number of open workshops grows. This can be expected in the case of Berlin, as the Maker Movement and collaboration and sharing culture in the city are growing stronger, fueled by the expanding community and hacking- or making-focused events, as all respondents of the survey pointed out.

\subsection{Next Steps}

The research presented revealed a number of interesting insights. However, it also shed light on some of the major gaps and challenges in understanding the connection between open workshops and sustainability. Particularly, there is lack of data on the concrete positive and negative effects of these places on economic development and conservation of the environment.

The methodology and the quantity of respondents in the present research are also rather limited. There had been a total of 98 co-creation spaces in Berlin identified during the research period, and their number continues to grow. Thus, in order to gain a broader perspective and more credible data many more of these spaces should be involved in the research. The survey itself should not be the only method of getting the data needed. Concrete numbers on economic value creation, social welfare enhancement, and waste and GHG emissions reduction should be gathered and analyzed to estimate and determine the quantitative contribution of open workshops to sustainability.

Finally, one should also assess the development trends and dynamics of collaborative innovation in open workshops in Germany and worldwide. These dynamics can be compared to the ones taking place in closed innovation processes within large corporations. Possible scenarios can be constructed to suggest how the current developments in this arena can be translated into the future. This analysis should indicate the areas where external support can be applied in order to channel the potential of open workshops into sustainable development.

\section{"There is no intentional contribution of co-creation spaces to sustainable development, but there are still indirect positive effects of these spaces."}

All in all, much more thorough research is needed to fully understand the connection between collaborative innovation in open workshops and sustainable development. And the next logical step will be to capitalize on the findings outlined in the current article, address the knowledge gaps identified herein, conduct data gathering and analysis on a broader scale by reaching many more open workshops, and then determine concrete points of correlation between open workshop activity and sustainable development in a city.

\section{Conclusions and recommendations}

The research on collaborative sustainability in open workshops conducted in the case of Berlin brought the following key results and conclusions. Firstly, The Maker Movement and its manifestation in co-creation spaces worldwide continue to grow as one of the global megatrends alongside sustainable development. Berlin is one of the European hotspots of these spaces, as it has a history of establishing the hacking and making culture and has nurtured a large and vibrant community of hackers and makers.

Secondly, the definition and classification of open workshop are still unclear, as each workshop is quite unique and different from others, even of the same type. However, it is still possible to define common features of open workshops, such as presence in a physical space, community-focused functioning of these places, promotion of collaborative relations and mutual trust among their members. Also one can classify all co-creation spaces into twelve types: co-working space, hackerspace, makerspace, maker faire, maker lab, fab lab, tech shop, specialized workshop, 3D printing café/hub, repair café/shop.

Thirdly, the existing literature on the topic of open workshops and sustainability suggests that there are both positive and negative implications of collaborative peer-based production in these workshops on sustainable development. The positive effects include potential reduction of GHG emissions and waste, increased resource efficiency, shift away from overconsumption and planned obsolescence of goods, promotion of sharing of tools and resources, integration of circular material flows and zero-waste principles into the production at open workshops, and reduction of environmental footprint from 
each product's creation and from the workshops themselves. Still, there are some negative implications mentioned in the theory as well. These concern mainly the possibility of overproduction and a higher waste generation rate, the large amount of energy needed to operate the machines in the workshops, and the potential still of GHG emissions caused by transportation of products over long distances.

Fourthly, empirical evidence suggests that open workshops are established and function not as sustainability-oriented economic agents. That is, nowadays there is no intentional direct contribution of co-creation spaces to sustainable development, at least not among the ones surveyed during the research. But there are still indirect positive effects of these spaces on different aspects of sustainability.

Fifthly, the contribution of open workshops to the economic side of sustainability is rather limited because such places are registered mostly as non-profit organizations and are not designed to be significantly profitable. Their economic contribution might be represented in the economic value of their products, but the research does not include this aspect as monitoring of these products is not performed by the workshops.

Sixthly, the low economic influence of open workshops is balanced by their significant implications for community engagement and social welfare. These places allow members of the local society to create their own goods in an efficient way with minimal costs, work on personal projects that have the potential to become profitable businesses, connect to networks of peers, and mutually improve knowledge and skills through sharing with each other.

Seventhly, the environmental responsibility of open workshops is mostly about minimizing waste generation by reusing, repurposing, and recycling of different waste streams. However, such resource efficiency is driven not by environmental concerns, but mainly by the fact that co-creation spaces place value on certain waste items as useful resources in their projects and in their space in general. Nevertheless, there is still potential for workshops to bring indirect contributions to better environmental management as a source of eco-innovations.

The research also identified some major gaps in knowledge on the topic of collaborative innovation and commons-based peer production in open workshops. Specifically, more data and analysis are needed on quantitative effects of open workshops and activities within them on different aspects of sustainability, on projects and innovations emerging from these places, and on their place in modern development trends and dynamics in comparison to a more traditional corporate innovation process.

\section{Remarks}

[1] The difference between hacking and making is that hacking presumes modifying an existing device to function better or serve a new, different purpose, while making is creating a device from scratch.

\section{References}

Anderson, C. (2012): Makers. The New Industrial Revolution. New York. Business Insider (2014): This Drivable Car Was Just 3D Printed In 44 Hours. Available on the internet at: www.businessinsider.com/3d-printedcar-2014-9

c-base (2014): About c-base. Available on the internet at: www.c-base.org/ cv50f

Cavalcanti, G. (2013): Is it a Hackerspace, Makerspace, TechShop, or FabLab? Make. Available on the internet at: www.makezine.com/2013/05/22/thedifference-between-hackerspaces-makerspaces-techshops-and-fablabs

FilaMaker (2014): Documentation and Support. Available on the internet at: www.filamaker.eu

Hackerspaces.org (2013): List of all hacker spaces. Available on the internet at: www.hackerspaces.org/wiki/Berlin

Iscenco, A. (2014): The Growth of Degrowth: Outcomes of the Degrowth 2014 Conference. Available on the internet at: www.blog.postwachstum.de/thegrowth-of-degrowth-outcomes-of-the-degrowth-2014-conference-20141016

Kostakis, V. et al. (2014): Production and governance in hackerspaces: A manifestation of Commons-based peer production in the physical realm? In: International Journal of Cultural Studies 1/19. Available on the internet at: www.ics.sagepub.com/content/early/2014/02/13/ 1367877913519310.abstract

MIT's Center for Bits and Atoms (2007): The Fab Charter. Available on the internet at: www.fab.cba.mit.edu/about/charter

Moilanen, J. (2012): Peer production communities survey 2011. Statistical Studies of Peer Production. Available on the internet at: www.surveys. peerproduction.net/2012/02/peer-production-communities-survey-2011

Lang, D. (2013): Zero to Maker. Learn (Just Enough) to Make (Just About) Anything. Maker Media, Inc. Available on the internet at: www.cdn.makezine.com/make/day-of-making/Zero_to_Maker.pdf

Lobo, R. (2011): Hackerspaces: from electrical to cultural resistance. International Symposium on Electronic Arts, Istanbul, September 2011.

Parker, E. (2013): In China, Lessons of a 'Hackerspace'. Do-it-yourself hubs are giving a boost to tinkerers and inventors. In: Wall Street Journal, 4. 10. 2013. Available on the internet at: www.online.wsj.com/news/ articles/SB10001424052702303722604579111253495145952

The Economist (2009): Triple bottom line. It consists of three Ps: profit, people and planet. November $17^{\text {th }}, 2009$. Available on the internet at: www.economist.com/node/14301663

Verbund Offener Werkstätten (2014): Werkstatt-Suche. Available on the internet at: www.offene-werkstaetten.org/werkstatt-suche

\section{AUTHOR + CONTACT}

Alexandr Iscenco is researcher at Moldovan Environmental Governance Academy (MEGA) focusing on the topics of collaborative environmental management and governance, open-source eco-innovations, and green entrepreneurship.

Moldovan Environmental Governance Academy (MEGA), 11 Moscova Street, MD-2068 Chisinau, Moldova. Phone: +37369 675157,

Email: hello@megageneration.com, Internet: www.megageneration.com 